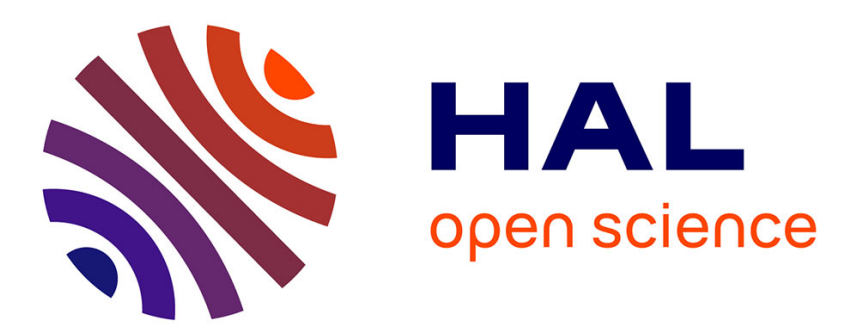

\title{
Kinetic theory of nucleation in multicomponent systems: An application of the thermodynamic extremum principle
}

\author{
Manon Bonvalet-Rolland, Thomas Philippe, John Ågren
}

\section{To cite this version:}

Manon Bonvalet-Rolland, Thomas Philippe, John Ågren. Kinetic theory of nucleation in multicomponent systems: An application of the thermodynamic extremum principle. Acta Materialia, 2019, 171, pp.1-7. 10.1016/j.actamat.2019.03.031 . hal-02413181

\section{HAL Id: hal-02413181 \\ https://hal.science/hal-02413181}

Submitted on 25 Aug 2021

HAL is a multi-disciplinary open access archive for the deposit and dissemination of scientific research documents, whether they are published or not. The documents may come from teaching and research institutions in France or abroad, or from public or private research centers.
L'archive ouverte pluridisciplinaire HAL, est destinée au dépôt et à la diffusion de documents scientifiques de niveau recherche, publiés ou non, émanant des établissements d'enseignement et de recherche français ou étrangers, des laboratoires publics ou privés. 


\title{
Kinetic theory of nucleation in multicomponent systems: an application of the thermodynamic extremum principle
}

\author{
Manon Bonvalet-Rolland ${ }^{\mathrm{a}}$, Thomas Philippe ${ }^{\mathrm{b}}$, John Ågren $^{\mathrm{a}}$ \\ ${ }^{a}$ Department of Materials Science and Engineering, KTH Royal Institute of Technology, \\ Stockholm, Sweden \\ ${ }^{b}$ Physique de la Matière Condensée, Ecole polytechnique, CNRS, 91120 Palaiseau, France
}

\begin{abstract}
Nucleation kinetics in a multicomponent supersaturated solid solution is examined. Attachment rate of atoms to a nucleus of a size close to the critical one is determined combining a thermodynamic extremum principle and the Fokker-Planck equation. Two limiting cases are examined; when bulk diffusion controls the nucleation kinetics and when the process is limited by the interfacial mobility. The mixed regime is also treated. Moreover, the growth law in multicomponent alloys is derived in the general case, when both mechanisms are considered. Additionally, the attachment rate is derived, in the classical framework, from a new macroscopic growth equations and the fundamental role of the interfacial mobility is examined. These new general expressions, for the attachment rates and the growth laws, determined either applying the thermodynamic extremum principle or derived from the classical formalism are found to be consistent.
\end{abstract}

Keywords: nucleation, thermodynamics, kinetics, extremum principle, multicomponent 


\section{Introduction}

The kinetics of precipitation in a supersaturated solid solution is of major concern in materials science. Indeed, it governs the microstructure and thus the material properties. The early stages of precipitation phenomena are often described in the framework of the classical nucleation theory (CNT) [1-4]. The stationary nucleation rate, i.e. the number of new nuclei per unit volume and unit time, in this formalism is expressed:

$$
J_{s t}=n Z \beta^{*} \exp \left(-\frac{W^{*}}{k T}\right)
$$

with $n$ the number of nucleation sites per unit volume, $Z$ the Zeldovich factor, $\beta^{*}$ the atomic attachment rate, $W^{*}$ the nucleation barrier, $k$ the Boltzman constant and $T$ the temperature. The above expression of the nucleation rate first derived by Zeldovich [3], accounts for the so-called regression effects, and holds for one dimensional nucleation theory, i.e. clusters are function of only one parameter, typically their size or the number of atoms they contains. Many refinements have been proposed [5-14], for instance, additional variables have been added to the description for a better understanding of the nucleation path, such as in binary systems, in focusing especially on the properties of pre- and post-critical clusters. Among the seminal theories is the classical work by Reiss [7] who proposed that the nucleation barrier is given by the minimum energy path along the nucleation landscape. Later, it has been shown that the flux direction could in fact be driven by both kinetics and thermodynamics $[8,9]$. This theory has been extended to multicomponent systems by Trinkaus [10]. Recently, a major improvement is due to Alekseechkin [11] who develops a general formalism, the multivariable theory, in which the nucleation rate is invariant with the space dimensionality and includes the one-dimensional theory, as it must. Since, nucleation is of fundamental interest for both the scientific community and industry, especially with the widespread availability of thermodynamic and kinetic databases, a quantitative description of nucleation applicable to real materials is much needed.

Despite technical progress in our understanding of nucleation, Eq. (1) remains used in most of the numerical models of nucleation and more generally of precipitation [15-20], because of its simplicity. Coupling such a model 
to a thermodynamic database allows for a quantitative description of nucleation thermodynamics, in computing accurately the quantities $W^{*}$ and $Z$. However, one of the challenging tasks when applying the CNT formalism is to express the atomic attachment rate $\beta^{*}$ properly. Here it is important to emphasize that depending on the space in which this coefficient is expressed, it will take another name. In the size space $\{r\}$ it is called the condensation rate, $\beta_{r}(r)$, whereas in the nucleus number of atoms space $\{N\}$ it is called the atomic attachment rate, $\beta_{N}(r)$. Obviously their unit are different and the conversion from one to another is easily made using [21]:

$$
\beta_{N}(r)=\left(\frac{4 \pi r^{2}}{v_{a t}}\right)^{2} \beta_{r}(r)
$$

with $v_{a t}$ the atomic volume. Eq. (2) accounts for the equivalence in the volume added whatever the space, i.e. $\{r\}$ or $\{N\}$. The atomic attachment rate involved in Eq. (1) is then obtained as $\beta^{*}=\beta_{N}\left(r^{*}\right)$. For the sake of clarity in the following the subscript $r$ is dropped out as expressions are exclusively derived in the $\{r\}$ space. Very often the expression of the atomic attachment rate used in modelling works has no proper thermodynamic and kinetic derivation [15, 22]. Expressions for binary alloys [5, 23] are sometimes expanded to multicomponent alloys and concern limit cases where either the reaction at interface or bulk volume diffusion limit the process. Another expression of $\beta^{*}$ has been recently proposed for multicomponent alloys by Philippe et al. [21] for bulk diffusion-limited nucleation in which the atomic attachment rate accounts for both diffusion and capillarity. The macroscopic growth law used in [21] to solve the Fokker-Planck equation is however only valid for low-supersaturated systems. In the literature several treatments of phase transformations based on a thermodynamic extremum principle (TEP) may be found [24-29]. The first use of such an approach to derive an attachment rate during nucleation was done by Svoboda et al. [24]. It is based on the fact that during nucleation, various irreversible processes dissipate the Gibbs energy until the system reaches equilibrium. The objective was to link the treatment of growth with the one of nucleation. In 1991, Svoboda et al. [25] proposed a mathematical treatment for applying their TEP. This mathematical tool does not add new physics but is aimed at deriving equations of motions of a thermodynamic system out of equilibrium when diffusion controls its evolution. Irreversible processes dissipate the total Gibbs energy of such system. Based on Onsagers principle [30] and Ziegler's TEP [31] the 
derivation in Svoboda's work allows to link the variation of the Gibbs energy dissipation function with respect to some characteristic parameters of the thermodynamic system and the variation of the total Gibbs energy with respect to the variation of the same characteristic parameters with time. It is actually like a balance equation describing the consumption of the available driving force for the transformation. In 2004, Svoboda et al. [26] applied this mathematical tool to the modelling of precipitation in multicomponent systems. They derived in an elegant way a growth and shrinkage equation based on diffusion fluxes in the matrix consuming the total Gibbs energy of the system. By doing so they also identified an atomic attachment rate for nucleation by comparing with both the growth law and attachment rate in the binary case. The obtained expression for the attachment rate in multicomponent alloys is largely used in precipitation softwares [32,33]. Nevertheless, this expression is not a proper derivation of the atomic attachment rate using a thermodynamic extremum principle. Application of the TEP was also described by Svoboda et al. [27] for non-steady grain growth. In 2006, Ågren and Hillert [34] compared the different extremum principles described in the literature and examined their derivation and assumptions. In 2014, Fisher et al. [29] summarized the limits of application of the different extremums principles and their potential for materials science. It was concluded that Onsager's and Ziegler's TEPs are valid if the kinetic equation for the dissipation can be expressed in a quadratic form of the fluxes. This hypothesis is fulfilled if the mobility of the interface and/or volume diffusion limit nucleation and growth.

In this work, Svoboda's TEP treatment is applied to precipitation limited by both bulk diffusion and interface mobility using a more general expression of the diffusive fluxes than the one used by Svoboda et al [26]. The condensation and atomic attachment rates are then obtained combining this treatment with the Fokker-Planck equation. It is also shown that applying TEP equations or a treatment involving the driving pressure, sometimes used in the literature, are strictly equivalent. Finally, a derivation of the macroscopic growth law without the use of the driving pressure is presented when both bulk diffusion and interface mobility limit precipitation. Combined with another treatment of the Fokker-Planck equation, the same expression of the condensation rate is again derived. The objective of this work is therefore to primarily derive a proper equation of the atomic attachment rate that can be directly used in the expression of the nucleation rate. However it is also 
highlighted that applying the TEP gives rise to the same results than more classical treatments which are in turn equivalent.

\section{Thermodynamic extremum principle}

In this work, only Onsager's extremum principle [30] and Ziegler's extremum principle [31] which are almost equivalent, Ziegler's one being the most general, are used. The reader is referred to the papers from Hillert and Agren [34] and Fischer et al. [29] for details on others extremal principles.

Thermodynamic extremum principles are mathematical tools to derive evolution equations of a thermodynamic system during an irreversible process [24-26, 29, 30, 34]. It allows to describe the evolution of the macroscopic parameters characterizing the system knowing the initial thermodynamic state of a closed thermodynamic system out of equilibrium. These macroscopic parameters are called characteristic parameters (CPs), $q_{i}$, and their evolution is described by time derivatives, $\dot{q}_{i}$. In the case of nucleation and growth of a phase $\beta$ in a supersaturated matrix $\alpha$, the generalized coordinates could be for instance the size of the precipitate, the concentrations of species or the number of nuclei.

In order to derive the master equations of TEPs available in the literature $[30,31]$, one starts with a fundamental equation from thermodynamics of irreversible processes where the rate of entropy production, $\dot{S}_{i p}$ due to internal processes in the system is expressed in the case of a closed system in isothermal and isobaric conditions:

$$
T \dot{S}_{i p}=\sum_{i} X_{i} J_{i}
$$

where $X_{i}$ is the driving force related to the flux $J_{i}$ of the internal process.

In an isobarothermal system the rate of entropy production is directly related to the variation of the Gibbs energy of the system as a function of time since $\dot{G}=-T \dot{S}_{i p}$. Thus

$$
-\dot{G}=\sum_{i} X_{i} J_{i}
$$


To make Eq. 4 useful for obtaining evolution equations in material sciences, a dissipation potential was introduced by Onsager at the same time as his reciprocal relations and kinetic phenomenological equations for diffusion fluxes [30]. These dissipation potentials are related to the driving force and fluxes dissipating the available driving force and were introduced to describe the way the driving force is used by the various irreversible processes. Coupled with these dissipation potentials, Onsager derived a Lagrangian function to be maximized to reproduce the principle of the least dissipation. This mathematical treatment led to the master equation in Onsager's TEP:

$$
\mathbf{X}=\frac{1}{T} \frac{1}{2} \frac{\partial Q}{\partial \mathbf{J}}
$$

Eq. 5 can be rewritten as a function of the characteristic parameters and characteristic parameter rates of the thermodynamic system. Then the evolution of the total Gibbs energy of the system, $G$, with respect to the generalized coordinates is proportional to the variation of the dissipation rate with respect to the generalized velocities $[24,25,29,34]$ :

$$
-\frac{\partial G}{\partial q_{i}}=\frac{1}{2} \frac{\partial Q}{\partial \dot{q}_{i}} .
$$

where the left-hand side is a generalized driving force. Eq. (6) is the master equation in Svoboda's TEP. It allows to find the path of the process and to derive the motion equations of the characteristic parameters during an irreversible process. Applications of the TEP may be found for several phase transformation problems in the literature [24-26, 28, 29].

As already mentioned, Eq. (1) is the expression of the stationary nucleation rate in a "one-dimensional space", i.e. a single parameter, the size of a nucleus, is considered. Likewise, most of the expressions for the condensation rate have been expressed in this size space. We work in the same space here. Then the generalized coordinate represents the size of the nucleus $r$ and the general velocity its growth or shrinkage rate $\dot{r}$ and Eq. (6) rewrites:

$$
-\frac{\partial G}{\partial r}=\frac{1}{2} \frac{\partial Q}{\partial \dot{r}}
$$


Solving Eq. (7) should then give information on the decomposition path in the $r$ space.

Ziegler generalized even more Onsager's TEP by taking into account in the Lagrangian expression to find the master equation the effect of constraints on the system. This could be a mass conservation constraint for example. It is worth mentionning that Ziegler's TEP is sometimes called the "thermodynamic extremum principle" and his formulation is used in this work.

When applying Onsager or Ziegler's TEP, the Gibbs energy of the system must be expressed as a function of the characteristic parameters. On their paper summarizing the use of thermodynamic extremal principles in materials science, Fischer et al. [29] described the equations issued from Ziegler's TEP which can be used in materials science. If the fluxes may be expressed linearly as a function of the CPs rates and the dissipation function in a quadratic form of the fluxes consuming the driving force, they showed that the dissipation function may be expressed as:

$$
Q=\sum_{i=1}^{N} \sum_{j=1}^{N} U_{i j}\left(q_{1}, q_{2}, \ldots, q_{N}\right) \dot{q}_{i} \dot{q}_{j}
$$

It can also be shown [29] that when applying Ziegler's TEP:

$$
\sum_{k=1}^{N} U_{i k} \dot{q}_{k}-\sum_{k=1}^{r} a_{i k} \beta_{k}=-\frac{\partial G}{\partial q_{i}}
$$

where the $\beta_{k}$ are the Lagrange multipliers taking into account the potential constraints and making the tool more general than Onsager's principle and allowing solving more complex material transformations. Without any considerations of constraints Eq. 9 rewrites

$$
\sum_{k=1}^{N} U_{i k} \dot{q}_{k}=-\frac{\partial G}{\partial q_{i}}
$$

and if one considers only the nucleus size $r$ as a characteristic parameter of 
the system and nucleation

$$
U(r) \dot{r}=-\frac{\partial W}{\partial r}
$$

where the matrix $U_{i k}$ becomes a scalar only function of $r$ and $W$ is the work of forming a nucleus of size $r$.

\section{Basic equations for describing nucleation}

In the phenomenological approach used for deriving the classical nucleation kinetic equations like Eq. (1), the evolution of the distribution function of clusters is described by the Fokker-Planck (FP) equation. Applied in the one-dimensional $r$ space, i.e. when the distribution function is a function of $r$ only, the FP equation is:

$$
\frac{\partial f(r)}{\partial t}=-\frac{\partial J(r)}{\partial r}
$$

where

$$
J(r)=-\beta(r) \frac{\partial f(r)}{\partial r}+f(r) \dot{r}
$$

$J(r)$ is the flux of clusters in the $r$ space, $f(r) \mathrm{d} r$ is the number of clusters of size in the range $r$ to $r+\mathrm{d} r, \beta(r)$ is the transition frequency of a cluster from $r$ to $r+d r$, the condensation rate, i.e. expressed in the size space $\{r\}$. The first term in Eq. (13) represents random fluctuations in size caused by thermal disorder and the second term, referred to as the drift, represents the thermodynamically driven growth or shrinkage of clusters. $\dot{r}(r)$ corresponds to the growth or shrinkage rate in the size space. The first term is always positive whereas the second term is negative for subcritical clusters and pos-

itive for supercritical clusters. In nucleation, where the crucial phenomena is growth of subcritical clusters, $\dot{r}(r)$ is always negative.

For a system in equilibrium, stable or metastable, the distribution of heterophase fluctuations is given by:

$$
f_{0}(r)=\text { const } \times \exp \left(-\frac{W(r)}{k T}\right)
$$


with subscript 0 denoting equilibrium. For the supercritical system we clearly have $\partial W / \partial r<0$, and Eq. 14 is not valid above the critical size.

For the equilibrium distribution $J(r)=0$ and $f(r)=f_{0}(r)$. Combination of Eqs. (12)-(14) then gives

$$
\dot{r}(r)=-\frac{\beta(r)}{k T} \frac{\partial W(r)}{\partial r}
$$

It implies that $\dot{r}(r)<0$ for subcritical sizes where $\partial W / \partial r>0$. Eq. (15) was also derived by Philippe et al. [21], but they considered a constant $\beta$. Eq. 15 is a key equation because it allows the evaluation of $\beta$ from the knowledge of $\dot{r}(r)$ and $W(r)$. It is interesting to note that Eq. 15 is similar to Eq. 11 highlighting the analogy between the phenomenological equation and the mathematical tool. It indicates that this tool contains the same physics as the classical equations and might be powerful when phenomenological equations are not available or with unknown parameters as $\beta(r)$.

We consider a case when the matrix $\alpha$ has a homogeneous content of species $j, x_{j}^{\alpha}$ and there is a driving force to precipitate a $\beta$ phase. Assuming spherical incompressible particles with isotropic interfacial energy $\sigma$ the work of formation of a spherical nucleus, or the Gibbs energy as a function of $r$ is then written as:

$$
W\left(r, x_{1}, \ldots, x_{n}\right)=G\left(r, x_{1}, \ldots, x_{n}\right)=-\frac{4 \pi}{3 V_{m}} r^{3} \Delta G_{m}+4 \pi r^{2} \sigma
$$

$\Delta G_{m}$ is the driving force normalized per mole of $\beta$ neglecting the capillarity effect. It is defined as positive when the $\beta$ phase is thermodynamically stable and is given by

$$
\Delta G_{m}=\sum_{i} x_{i}^{\beta} \mu_{i}^{\alpha}\left(x_{j}^{\alpha}\right)-G_{m}^{\beta}
$$

$V_{m}$ is the molar volume. The chemical potentials $\mu_{j}^{\alpha}$ are evaluated for the actual matrix composition $x_{j}^{\alpha}$ which at every instant will be approximated as homogeneous but may vary in time. The composition of $\beta, x_{j}^{\beta}$, is obtained from a multidimensional tangent plane to the $\beta$ Gibbs-energy curve parallel to the tangent plane of $\alpha$ for the matrix composition under consideration. Another way of expressing this is to say that $\Delta G_{m}$ (positive or negative) is 
the addition to the molar Gibbs energy of the $\beta$ phase that would cause the two phases to be in equilibrium. Actually, this is how the driving force is calculated in Thermo-Calc [35].

The generalized driving force $-\partial G / \partial r$, right-hand side in Eq. 11, is obtained from Eq. 16 as

$$
-\frac{\partial G}{\partial r}=\frac{4 \pi}{V_{m}} r^{2} \Delta G_{m}-8 \pi r \sigma
$$

The capillarity effect thus reduces the generalized driving force for formation of a particle with radius $r$ and there is a critical value $r^{*}$ where it vanishes, the critical size. As mentioned this particle size represents an unstable equilibrium; if the particle is smaller it will dissolve and if it is bigger it will grow. The critical size is obtained from Eq.18 as

$$
r^{*}=2 \sigma V_{m} / \Delta G_{m}
$$

If the Gibbs energy of the precipitate phase is always higher than that of the matrix the driving force $\Delta G_{m}$ will always be negative and Eq. (19) will result in a negative critical radius, i.e. the particle could never grow but will always dissolve. According to the mean-field approximation an assembly of $\beta$ particles in a homogeneous $\alpha$ matrix is considered and we may introduce the critical size from Eq. (19) and rephrase Eq. 18 into

$$
-\frac{\partial G}{\partial r}=8 \pi \sigma r^{2}\left[\frac{1}{r^{*}}-\frac{1}{r}\right]
$$

We have now discussed the right-hand side in Eq. 11 and in the next section we shall turn to the left-hand side, i.e. the dissipation. Indeed in order to identify $U(r)$ and in turn express $\beta$, an expression of the dissipation rate $Q$ is needed, see Eqs. (8), (11) and (15). 


\section{Application of the TEP to nucleation and growth}

In this section we first directly apply the equations introduced in section 1 from the TEP's treatment and evaluate the dissipation rate $Q$ as a function of the characteristic parameters rates, i.e. the growth rate in $1 \mathrm{D}$. However, the same type of equations, but involving the so-called driving pressure, are sometimes also applied in the literature to derive the growth rate. Therefore to highlight the strict equivalence between the two treatments, we show in the second part how one can obtain the dissipation rate from the driving pressure.

To express $Q$, one should identify the various irreversible processes dissipating the available driving force. That would make possible the identification of the matrix $\mathbf{U}$ needed to obtain Eq. 9. The dissipation function $Q$ is actually the variation of the Gibbs energy as a function of time, $Q=\dot{G}$. In this work where the goal is to emphasize the usefulness of the TEP for finding the evolution equation during nucleation, we first consider that the internal process consuming the available driving force is unique and is the diffusion of atoms in the matrix. In that case:

$$
Q^{t o t}=Q^{d i f f}=-\int \mathbf{J}^{\mathbf{T}} \nabla \boldsymbol{\Phi} \mathrm{d} V
$$

with $\mathbf{J}^{\mathbf{T}}$ the transpose of the columnar diffusion fluxes vector $\mathbf{J}$, and $\nabla \boldsymbol{\Phi}$ the chemical potential gradient which is the driving force for diffusion, where $\Phi_{j}$ is the diffusion potential e.g. in the number-fixed frame of reference $\Phi_{j}=\mu_{j}-\mu_{N}$ when $N$ is chosen as reference element. Assuming spherical symmetry and a flux expressed only in the radial direction, Eq. 21 may be written approximately as:

$$
Q^{d i f f} \approx-4 \pi r^{3} \mathbf{J}^{\mathbf{T}} \nabla \boldsymbol{\Phi} r
$$


The diffusion fluxes are [36]

$$
J_{i}^{\alpha}=-\frac{1}{V_{m}} \sum_{j}^{N-1} L_{i j}^{\alpha} \nabla \Phi_{j}
$$

$L_{i j}^{\alpha}$ is the phenomenological matrix in the frame of reference under consideration. In order to simplify the notation we use matrix vector notation and drop the index $\alpha$ and write Eq. (23) as

$$
\mathbf{J}=-\frac{1}{V_{m}} \mathbf{L} \nabla \boldsymbol{\Phi}
$$

L is the symmetric $(N-1) \times(N-1)$ matrix with the coefficients $L_{i j}^{\alpha}$. It should be emphasized that we can let $\mathbf{L}$ be a $N \times N$ matrix and the treatment will then cover also the lattice-fixed frame of reference. $\mathbf{L}$ generally depends on composition. Again assuming a simple spherical symmetry with variation only in the radial direction $\zeta$ we have $\nabla \boldsymbol{\Phi}=\partial \boldsymbol{\Phi} / \partial \zeta$. Compared to Svoboda's treatment [26] Eq. (24) is more general due to the use of the phenomenological equation for diffusion.

The flux balance equation in dyadic notation is

$$
\frac{\dot{r}}{V_{m}} \Delta \mathbf{x}=-\mathbf{J}
$$

where $\Delta \mathbf{x}$ is a columnar vector with elements $x_{i}^{\beta}-x_{i}^{\alpha}$.

Combining Eqs. (22), (24) and (25)

$$
Q^{d i f f}=\frac{4 \pi r^{3}}{V_{m}} \Delta \mathbf{x}^{\mathbf{T}} \mathbf{L}^{-1} \boldsymbol{\Delta} \mathbf{x} \dot{r}^{2}
$$

Comparing with the TEP equation Eq. (8), we can identify the matrix $U_{i j}$ that is a single coefficient $U(r)$ in a 1D-treatment:

$$
U(r)=\frac{4 \pi r^{3}}{V_{m}} \Delta \mathbf{x}^{\mathbf{T}} \mathbf{L}^{-1} \Delta \mathbf{x} .
$$

It is worth mentioning that this result could also have been obtained by introducing the driving pressure $P^{t o t}$ as it is shown below. Nevertheless using 
the driving pressure to derive $\dot{r}$ involves both the use of the thermodynamics of irreversible processes and the use of phenomenological kinetic equations for diffusion fluxes, i.e. the same basic equations than for deriving the TEP tool equations. Indeed Eq. 21 derived with the expression of the rate of entropy production may be rewritten in terms of driving pressure introducing that the driving pressure for a particle of radius $r$ to grow is expressed from $\Delta G_{m}$ and subtracting the retarding effect of capillarity.

$$
P^{t o t}(r)=\frac{\Delta G_{m}}{V_{m}}-\frac{2 \sigma}{r}
$$

A positive value yields growth whereas a negative shrinkage. The value 0 occurring at the critical size represents the unstable (critical) equilibrium with the matrix.

We can also express $\dot{G}$ as

$$
\dot{G}=\frac{\partial G}{\partial r} \dot{r}
$$

when only one characteristic parameter is considered. Therefore according to Eq. (18) combined with Eq. (28) we obtain:

$$
\dot{G}=-4 \pi r^{2} P^{t o t} \dot{r}
$$

Therefore relating Eq. (30) to Eq. (26) we obtain:

$$
P^{t o t} \dot{r}=\frac{r}{V_{m}} \dot{r}^{2} \Delta \mathbf{x}^{\mathbf{T}} \mathbf{L}^{-1} \Delta \mathbf{x}
$$

that may be developed to obtain an expression similar to Eq. (26), the difference being that Eq. (31) gives the dissipation per area of particle matrix interface.

Eq. (26) and Eq. (31) are positively quadratic forms of $\dot{r}$ as it must when a precipitate is only defined by its size. Including also the dissipation by interface friction, Eq. (31) becomes

$$
Q^{t o t}=\frac{4 \pi r^{3}}{V_{m}} v^{2} \boldsymbol{\Delta} \mathbf{x}^{\mathbf{T}} \mathbf{L}^{-1} \boldsymbol{\Delta} \mathbf{x}+\frac{4 \pi r^{2}}{M^{i n t}} v^{2}
$$


with $M^{\text {int }}$ the interface mobility.

When using the driving pressure expressions, Eq. (28) and Eq. (31), it is possible to derive the growth rate as:

$$
\dot{r}=\frac{1}{r} \frac{\Delta G_{m}(r)}{\left(\frac{V_{m}}{M^{\text {int }} r}+\boldsymbol{\Delta} \mathbf{x}^{\mathbf{T}} \mathbf{L}^{-1} \boldsymbol{\Delta} \mathbf{x}\right)}=\frac{1}{r} \frac{2 \sigma V_{m}}{\left(\frac{V_{m}}{M^{\text {int }} r}+\Delta \mathbf{x}^{\mathbf{T}} \mathbf{L}^{-1} \boldsymbol{\Delta} \mathbf{x}\right)}\left[\frac{1}{r^{*}}-\frac{1}{r}\right]
$$

The expression without interface friction:

$$
\dot{r}=\frac{1}{r} \frac{\Delta G_{m}-2 \sigma V_{m} / r}{\Delta \mathbf{x}^{\mathbf{T}} \mathbf{L}^{-1} \boldsymbol{\Delta} \mathbf{x}}=\frac{1}{r} \frac{2 \sigma V_{m}}{\Delta \mathbf{x}^{\mathbf{T}} \mathbf{L}^{-1} \boldsymbol{\Delta} \mathbf{x}}\left[\frac{1}{r^{*}}-\frac{1}{r}\right]
$$

i.e. when $M^{\text {int }} \rightarrow \infty$, may be directly obtained combining Eqs. (11), (15), (20) and (27) and is close to the one derived by Philippe and Voorhees [37] for low supersaturated multicomponent alloys:

$$
\dot{r}=\frac{1}{r} \frac{1}{\Delta \overline{\mathbf{x}}^{\mathrm{T}} \overline{\mathbf{L}}^{-1} \boldsymbol{\Delta} \overline{\mathbf{x}}}\left[\Delta \overline{\mathbf{x}}^{\mathbf{T}} \mathbf{G}^{\alpha} \Delta \mathbf{x}^{\alpha}-\frac{2 \sigma V_{m}}{r}\right]
$$

It was indeed shown by Philippe et al. [38] that the driving force for nucleation in low supersaturated alloy is $\Delta G_{m}=\Delta \overline{\mathbf{x}}^{\mathbf{T}} \mathbf{G}^{\alpha} \Delta \mathbf{x}^{\alpha}$ with $\mathbf{G}^{\alpha}$ the Hessian of the Gibbs energy density of $\alpha$ evaluated at equilibrium concentrations, $\overline{\mathbf{L}}$ is the mobility matrix of size $(N-1) \times(N-1)$ such as $\mathbf{D}=\overline{\mathbf{L}} \mathbf{G}^{\alpha}$ with $\mathbf{D}$ the interdiffusion matrix in $\alpha$, as $\mathbf{G}^{\alpha}$ both $\overline{\mathbf{L}}$ and $\mathbf{D}$ are evaluated at equilibrium concentrations. The matrix $\overline{\mathbf{L}}$ contains both kinetic and thermodynamic information. $\Delta \mathbf{x}^{\alpha}$ is the supersaturation tie-line, i.e. $x_{j}^{\alpha}-\bar{x}_{j}^{\alpha}$ with $\Delta \mathbf{x}^{\alpha}$ the equilibrium composition. $\Delta \overline{\mathbf{x}}^{\mathbf{T}}$ is the equilibrium tie-line vector, $\bar{x}_{j}^{\beta}-\bar{x}_{j}^{\alpha}$. In the limit $M^{\text {int }} \rightarrow \infty$, Eq. (34) and Eq. (35) are then equivalent if

$$
\Delta \overline{\mathrm{x}}^{\mathrm{T}} \overline{\mathrm{L}}^{-1} \Delta \overline{\mathrm{x}}=\Delta \mathrm{x}^{\mathrm{T}} \mathbf{L}^{-1} \Delta \mathrm{x}
$$

that holds for low-supersaturated systems.

\section{The atomic attachment rate $\beta^{*}$}

Let us now use the expression for $U(r)$ derived in the previous section, Eq. (27), together with one of the equation in the TEP treatment, Eq. (11), 
and the FP equation, Eq. (15). When comparing Eqs. (11) and (15) , $\beta(r)$ writes:

$$
\beta(r)=\frac{k T}{U(r)}
$$

When replacing $U(r)$ by its expression, Eq. (27), in the case of diffusionlimited nucleation, we finally obtain:

$$
\beta(r)=\frac{k T V_{m}}{4 \pi r^{3} \Delta \mathbf{x}^{\mathbf{T}} \mathbf{L}^{-1} \boldsymbol{\Delta} \mathbf{x}}
$$

It is worth mentioning that the relation Eq. (37) between $\beta$ and $U$ was also proposed by Svoboda et al. in [24] on their work on cavity nucleation and could also be found when developing equations in [25]. Nevertheless, because the expressions of the dissipation rate were different, the same relation does not hold for $\beta$ itself, i.e. Eq. (38). The derivation in our work is much more general because based on Eq. (24) which is the most general equation describing diffusive fluxes. Besides, due to the increasing availability of kinetic databases, Eq. (38) may be solved for fixed thermodynamic conditions. The tie-line is the one found when studying precipitation kinetics [39].

Eq. (38) expressed for the critical radius is similar to the expression derived by Philippe et al. [21] $\beta^{*}=k T V_{m} /\left(4 \pi r^{* 3} \boldsymbol{\Delta} \mathbf{x}^{\mathbf{T}} \mathbf{L}^{-1} \boldsymbol{\Delta} \mathbf{x}\right)$ when Eq. (36) holds, i.e. for low-supersaturated system, and for the critical radius. The expression in [21] is valid when the concentration variable drops out of consideration. They showed using the formalism of the multivariable theory [11] that it is the case when the curvature of the precipitate phase Gibbs energy is very large.

If we now express $U(r)$ when interface friction and diffusion are both considered as possible rate-limiting mechanisms, the condensation rate becomes:

$$
\beta(r)=\frac{k T V_{m}}{4 \pi r^{2}}\left(r \Delta \mathbf{x}^{\mathbf{T}} \mathbf{L}^{-1} \boldsymbol{\Delta} \mathbf{x}+\frac{V_{m}}{M_{\text {int }}}\right)^{-1}
$$

$\beta^{*}$ is subsequently obtained converting Eq. (39) in the appropriate $\{N\}$ space with Eq. (2):

$$
\beta^{*}=\beta_{N}\left(r^{*}\right)=\frac{4 \pi r^{* 2} k T V_{m}}{v_{a t}^{2}}\left(r \Delta \mathbf{x}^{\mathbf{T}} \mathbf{L}^{-1} \mathbf{\Delta} \mathbf{x}+\frac{V_{m}}{M_{i n t}}\right)^{-1}
$$


The expression derived in this work is function of the nucleus size and accounts for capillarity and diffusion-flux couplings with the matrix $\mathbf{L}$.

\section{Kinetic theory of nucleation}

In this part we show that the expression of $\beta^{*}$ obtained in the previous section applying the TEP equations may also be derived employing macroscopic growth law and another form of the Fokker-Planck equation. It emphasizes another route for solving the Fokker-Planck equation and expressing the atomic attachment rate but does not contain another physics than what has been derived in the previous sections.

\subsection{The role of the interfacial mobility in nucleation}

In the general formalism introduced by Alekseechkin [11], the kinetics coefficient of the nucleation theory (diffusivities in the Fokker-Planck equation) are determined from the macroscopic equations of motion of a nucleus in the considered space. Very often macroscopic thermodynamics is employed to describe the work of nucleus formation, but the kinetic coefficients are deduced from microscopic considerations such as the kinetic processes on the interface. In contrast, in the following, the diffusivities and thus the nucleation rate are determined by macroscopic parameters, and such approach can therefore be seen as fully macroscopic. Let us first examine the case where growth is controlled by the intrinsic mobility of the interface and not by diffusion. We assume that the interface linearly responds to a difference in energy, thus, we write for the normal interface velocity:

$$
v=\frac{M^{i n t}}{V_{m}} \Delta G
$$

where $M^{\text {int }}$ is the intrinsic mobility of the interface (in $m^{4} J^{-1} s^{-1}$ ) and $\Delta G$ is given by

$$
\Delta G=\sum_{j=1}^{N} x_{j}^{\beta, i}\left(\mu_{j}^{\alpha}\left(x_{j}^{\alpha, i}, P^{\alpha}\right)-\mu_{j}^{\beta}\left(x_{j}^{\beta, i}, P^{\beta}\right)\right)
$$


$\mu_{j}^{\alpha}$ is the chemical potential in the $\alpha$ phase of element $j, \mu_{j}^{\beta}$ in the $\beta$ phase. As it must, local equilibrium at the interface systematically implies no interface motion. $x_{j}^{\alpha, i}$ and $x_{j}^{\beta, i}$ are the compositions on both sides of the interface for a precipitate of radius $r$. We assume mechanical equilibrium

$$
P^{\beta}=P^{\alpha}+\frac{2 \sigma}{r}
$$

and use

$$
\mu_{j}^{\beta}\left(P^{\beta}\right)=\mu_{j}^{\beta}\left(P^{\alpha}\right)+V_{j}^{\beta}\left(P^{\beta}-P^{\alpha}\right)
$$

with $V_{j}^{\beta}$ the partial molar volume. Observing that

$$
\sum_{j=1}^{N-1} x_{j}^{\beta, i}\left(\mu_{j}^{\alpha}\left(x_{j}^{\alpha, i}, P^{\alpha}\right)-\mu_{j}^{\beta}\left(x_{j}^{\beta, i}, P^{\alpha}\right)\right)=\Delta G_{m}
$$

as we consider that both the precipitates and the matrix are homogeneous in composition, Eq. (41) writes

$$
\dot{r}=2 \sigma M^{\text {int }}\left(\frac{1}{r^{*}}-\frac{1}{r}\right)
$$

Thus, a precipitate, with a composition $x_{j}^{\alpha, i}$ at its interface, is in equilibrium $(v=0)$ if its size is $r^{*}$, which is set by the matrix composition $x_{j}^{\alpha, i}$. If its actual size $r$ is smaller, the precipitate shrinks, or grows when $r>r^{*}$. Then, we use the same approach detailed in [21], the work of formation of a nucleus $W(r)$ is represented as a quadratic form near its saddle point ${ }^{*} c$ :

$$
W=W^{*}+\frac{1}{2} H_{r r}\left(r-r^{*}\right)^{2}
$$

where

$$
H_{r r}=\frac{\partial^{2} W}{\partial r^{2}}
$$

and $W^{*}=W\left(r^{*}\right)$ is the nucleation barrier. Assuming a constant $\beta$, Eq. (15) can be expressed as

$$
\dot{r}=-\frac{D_{r}}{k T}\left(r-r^{*}\right)
$$

with $D_{r}=\beta H_{r r}$ (not to be confused with the interdiffusion matrix). Eq. (49) is a motion equation in the size space. Therefore, expanding the macroscopic 
growth equation Eq. (46) around the critical size allows for the determination of the coefficient $D_{r}$ and then the condensation rate in the size space $\beta_{r}$ for interface-limited growth regime. Using $H_{r r}=-8 \pi \sigma[21]$, we find for $\beta_{r}$ (in $\left.m^{2} / s\right)$ :

$$
\beta_{r}=\frac{k T M^{i n t}}{4 \pi r^{* 2}}
$$

or in the n-space (with $\mathrm{n}$ the number of atoms in the cluster)

$$
\beta_{n}=\frac{k T M^{i n t}}{v_{a t}^{2}} 4 \pi r^{* 2}
$$

with $v_{a t}$ the atomic volume and where we have used $\beta_{r} / \beta_{n}=\left(4 \pi r_{c}^{2}\right)^{2} / v_{a t}^{2}[21]$. The size dependence of $\beta_{n}$ (in $s^{-1}$ ) is analogous to the well-known result for binary [3], but for a different physics:

$$
\beta^{*}=\frac{D x^{\alpha}}{a^{2}} 4 \pi r^{* 2}
$$

when growth is controlled by the jump frequency of solute atoms, $D$ is the interdiffusion coefficient, in multicomponent the slower diffuser is often used as a good approximation. A jump frequency $\Gamma$ is also used instead of $D$. However, our result (Eq. (50) or Eq. (51)), in case growth controlled by the interface response to a thermodynamic disequilibrium, contains the interfacial mobility, $M^{i n t}$, as it must. A similar expression has been derived in [40] but that contains a thermodynamic term and describes growth of supercritical clusters (far enough from the critical size).

\subsection{General case}

The growth equation, Eq. (25), in multicomponent system, can be written in dyadic notation using diffusivities $D$ as

$$
\boldsymbol{\Delta} \overline{\mathbf{x}} \frac{\mathrm{d} r}{\mathrm{~d} t}=\frac{\mathbf{D}}{r}\left[\Delta \mathbf{x}^{\alpha}-\Delta \mathbf{x}^{\alpha, i}\right]
$$

where $\Delta \overline{\mathrm{x}}$ is the equilibrium tie-line vector, $\bar{x}_{j}^{\beta}-\bar{x}_{j}^{\alpha} ; \Delta \mathrm{x}^{\alpha}$ is the supersaturation tie-line, i.e. $x_{j}^{\alpha}-\bar{x}_{j}^{\alpha} \cdot \Delta \mathrm{x}^{\alpha, i}$ gives the actual composition at the interface 
of a precipitate, $x_{j}^{\alpha, i}-\bar{x}_{j}^{\alpha}$. In the limit of low supersaturation $\Delta \overline{\mathbf{x}}=\Delta \mathbf{x}$. As we are interested by the general case and contrary to Eq. (35), we do not assume local equilibrium, consequently the interfacial compositions are unknown. This disequilibrium makes the interface to move at a velocity given by Eq. (46). Still in the limit of low supersaturation, it can be shown that

$$
v=\dot{r}=\frac{M^{i n t}}{V_{m}} \Delta \overline{\mathbf{x}}^{\mathbf{T}} \mathbf{G}^{\alpha}\left(\Delta \mathbf{x}^{\alpha, i}-\Delta \mathbf{x}^{\alpha, r}\right)
$$

with $\Delta \mathbf{x}^{\alpha, r}=x_{j}^{\alpha, r}-\bar{x}_{j}^{\alpha}$ where $x_{j}^{\alpha, r}$ is the composition at the interface of a precipitate or radius $\mathrm{r}$ in equilibrium with the matrix, i.e. the composition given by the Gibbs-Thomson equation [37]. Interestingly, we find that the interface linearly responds to the difference in composition between the actual interfacial composition and its value at equilibrium. In multicomponent alloys, a precipitate in equilibrium with the matrix satisfies [37]:

$$
\boldsymbol{\Delta} \mathbf{x}^{\alpha, r}=\boldsymbol{\Delta} \mathbf{x}^{\alpha}-\frac{\mathbf{D}^{-1} \boldsymbol{\Delta} \overline{\mathbf{C}}}{\boldsymbol{\Delta} \overline{\mathbf{x}}^{\mathbf{T}} \overline{\mathbf{L}}^{-1} \boldsymbol{\Delta} \overline{\mathbf{x}}}\left[\boldsymbol{\Delta} \overline{\mathbf{x}}^{\mathbf{T}} \mathbf{G}^{\alpha} \boldsymbol{\Delta} \mathbf{x}^{\alpha}-\frac{2 \sigma V_{m}}{r}\right]
$$

as the composition at the interface is not only set by the capillary effect, contrary to binaries, but is also dependent on the diffusion process. Combining Eqs. (53)-(55) gives:

$$
\dot{r}=\frac{2 \sigma V_{m}}{r}\left(\frac{1}{r^{*}}-\frac{1}{r}\right)\left(\Delta \overline{\mathbf{x}}^{\mathbf{T}} \overline{\mathbf{L}}^{-1} \Delta \overline{\mathbf{x}}+\frac{V_{m}}{r M^{\text {int }}}\right)^{-1}
$$

which is analogous to Eq. (33). Expanding the growth law near the critical size and using Eq. (49), we find the following general expression for the condensation rate:

$$
\beta_{r}=\frac{k T V_{m}}{4 \pi r^{* 2}}\left(r^{*} \Delta \overline{\mathbf{x}}^{\mathbf{T}} \overline{\mathbf{L}}^{-1} \boldsymbol{\Delta} \overline{\mathbf{x}}+\frac{V_{m}}{M^{i n t}}\right)^{-1}
$$

This new expression contains both diffusion and the effect of the interfacial mobility. When the last effect is negligible, i.e. when the interfaces response to a thermodynamic disequilibrium is fast enough $\left(M^{\text {int }} \rightarrow \infty\right)$, as compared with solute diffusion, the bulk diffusion-controlled regime is recovered [21]:

$$
\beta_{r}=\frac{k T V_{m}}{4 \pi r^{* 3} \Delta \overline{\mathbf{x}}^{\mathbf{T}} \overline{\mathbf{L}}^{-1} \Delta \overline{\mathbf{x}}}
$$


and for low interfacial mobility, the interface-limited regime, Eq. (50), is recovered. Both results are consistent with the TEPs expressions (Eq. (39)).

\section{Conclusion}

In the present work, we have derived using Svoboda's TEP a new expression of the atomic attachment rate for both bulk diffusion and interface reaction limited nucleation and a new expression for the growth rate in multicomponent alloys. We show that this mathematical tool is actually similar in its physical description to the classical expression with the driving pressure used in previous works. We have also performed the analysis in a more classical framework, combining macroscopic equations for growth and nucleation theory. We show that both approaches contain the same physics and are consistent. When considering the same limiting mechanisms during nucleation and growth, TEP mathematical treatment may be a useful tool to derive motion equations of the characteristic parameters of the thermodynamic systems. When modelling precipitation, it is thus interesting because it allows being consistent in describing nucleation, growth and coarsening. Knowledge of the kinetic phenomenological coefficients allows for a quantitative calculation of the atomic attachment rate and thus of the nucleation rate. Those new expressions can be used in multicomponent alloys and accounts for both thermodynamic and kinetic information, as it must. It would be of a major interest to extend the TEP treatment in a multivariable space to consider the influence of the nucleus concentration on the nucleation process.

\section{Acknowledgement}

Two authors, MB and JA, want to acknowledge the FraMat project supported by VINNOVA, the Swedish Governmental Agency for Innovation Systems and Swedish industry. 


\section{References}

[1] M. Volmer and 1885-1965, Kinetik der phasenbildung. Steinkopff, Dresden/Leipzig, 1939.

[2] R. Becker and W. Dring, "Kinetische Behandlung der Keimbildung in bersttigten Dmpfen," Annalen der Physik, vol. 416, no. 8, pp. 719-752, 1935.

[3] J. Zeldovich, "Theory of nucleation and condensation," Soviet Phys.JETP, vol. 12, p. 525, 1942.

[4] Y. Frenkel, Kinetic theory of liquids. Oxford: Oxford University Press, 1946.

[5] J. Feder, K. C. Russell, J. Lothe, and G. M. Pound, "Homogeneous nucleation and growth of droplets in vapours," Advances in Physics, vol. 15, pp. 111-178, Jan. 1966.

[6] K. C. Russell, "Nucleation in solids: The induction and steady state effects," Advances in Colloid and Interface Science, vol. 13, pp. 205318, Sept. 1980.

[7] H. Reiss, "The Kinetics of Phase Transitions in Binary Systems," The Journal of Chemical Physics, vol. 18, pp. 840-848, June 1950.

[8] D. Stauffer, "Kinetic theory of two-component (hetero-molecular) nucleation and condensation," Journal of Aerosol Science, vol. 7, pp. 319-333, July 1976.

[9] G. Wilemski, "Binary nucleation kinetics. IV. Directional properties and cluster concentrations at the saddle point," The Journal of Chemical Physics, vol. 110, pp. 6451-6457, Mar. 1999.

[10] H. Trinkaus, "Theory of the nucleation of multicomponent precipitates," Physical Review B, vol. 27, pp. 7372-7378, June 1983.

[11] N. V. Alekseechkin, "Multivariable kinetic theory of the first order phase transitions," The Journal of Chemical Physics, vol. 124, p. 124512, Mar. 2006. 
[12] B. E. Wyslouzil and G. Wilemski, "Binary nucleation kinetics. II. Numerical solution of the birthdeath equations," The Journal of Chemical Physics, vol. 103, pp. 1137-1151, July 1995.

[13] B. E. Wyslouzil and G. Wilemski, "Binary nucleation kinetics. III. Transient behavior and time lags," The Journal of Chemical Physics, vol. 105, pp. 1090-1100, July 1996.

[14] S. P. Fisenko and G. Wilemski, "Kinetics of binary nucleation of vapors in size and composition space," Physical Review. E, Statistical, Nonlinear, and Soft Matter Physics, vol. 70, p. 056119, Nov. 2004.

[15] M. Bonvalet, T. Philippe, X. Sauvage, and D. Blavette, "Modeling of precipitation kinetics in multicomponent systems: Application to model superalloys," Acta Materialia, vol. 100, pp. 169-177, Nov. 2015.

[16] M. Perez, M. Dumont, and D. Acevedo-Reyes, "Implementation of classical nucleation and growth theories for precipitation," Acta Materialia, vol. 56, pp. 2119-2132, May 2008.

[17] M. Serriere, C.-A. Gandin, E. Gautier, P. Archambault, and M. Dehmas, "Modeling of Precipitation Coupled with Thermodynamic Calculations," Materials Science Forum, 2002.

[18] P. Maugis and M. Goun, "Kinetics of vanadium carbonitride precipitation in steel: A computer model," Acta Materialia, vol. 53, pp. 33593367, July 2005.

[19] J. D. Robson and P. B. Prangnell, "Dispersoid precipitation and process modelling in zirconium containing commercial aluminium alloys," Acta Materialia, vol. 49, pp. 599-613, Feb. 2001.

[20] J. D. Robson, "A new model for prediction of dispersoid precipitation in aluminium alloys containing zirconium and scandium," Acta Materialia, vol. 52, pp. 1409-1421, Apr. 2004.

[21] T. Philippe, M. Bonvalet, and D. Blavette, "Kinetic theory of diffusionlimited nucleation," The Journal of Chemical Physics, vol. 144, p. 204501, May 2016. 
[22] L. Rougier, A. Jacot, C.-A. Gandin, P. D. Napoli, P.-Y. Thry, D. Ponsen, and V. Jaquet, "Numerical simulation of precipitation in multicomponent Ni-base alloys," Acta Materialia, vol. 61, pp. 6396-6405, Oct. 2013.

[23] G. Martin, "The theories of unmixing kinetics of solid solutions," Tech. Rep. CEA-CONF-4776, CEA Centre d'Etudes Nucleaires de Saclay, 1979.

[24] J. Svoboda, I. Turek, and V. Sklenika, "Unified thermodynamic treatment of cavity nucleation and growth in high temperature creep," Acta Metallurgica et Materialia, vol. 38, pp. 573-580, Apr. 1990.

[25] J. Svoboda and I. Turek, "On diffusion-controlled evolution of closed solid-state thermodynamic systems at constant temperature and pressure," Philosophical Magazine B, vol. 64, pp. 749-759, Dec. 1991.

[26] J. Svoboda, F. D. Fischer, P. Fratzl, and E. Kozeschnik, "Modelling of kinetics in multi-component multi-phase systems with spherical precipitates: I: Theory," Materials Science and Engineering: A, vol. 385, pp. 166-174, Nov. 2004.

[27] J. Svoboda and F. D. Fischer, "A new approach to modelling of nonsteady grain growth," Acta Materialia, vol. 55, pp. 4467-4474, Aug. 2007.

[28] F. D. Fischer, J. Svoboda, and P. Fratzl, "A thermodynamic approach to grain growth and coarsening," Philosophical Magazine, vol. 83, pp. 10751093, Jan. 2003.

[29] F. D. Fischer, J. Svoboda, and H. Petryk, "Thermodynamic extremal principles for irreversible processes in materials science," Acta Materialia, vol. 67, pp. 1-20, Apr. 2014.

[30] L. Onsager, "Reciprocal Relations in Irreversible Processes. I.," Physical Review, vol. 37, pp. 405-426, Feb. 1931.

[31] H. ZIEGLER, "Some extremum principles in irreversible thermodynamics, with application to continuum mechanics," Progress in Solid Mechanics, Vol. 4, pp. 93-193, 1963. 
[32] "TC-PRISMA Users Guide and Examples, Thermo-Calc Software AB, Stockholm, (2015).."

[33] E. KOZESCHNIK, "MatCalc-A Simulation Tool for Multicomponent Thermodynamics, Diffusion and Phase Transformations," Mathematical Modelling of Weld Phenomena5, vol. 349, 2001.

[34] M. Hillert and J. gren, "Extremum principles for irreversible processes," Acta Materialia, vol. 54, pp. 2063-2066, May 2006.

[35] J.-O. Andersson, T. Helander, L. Hglund, P. Shi, and B. Sundman, "Thermo-Calc \& DICTRA, computational tools for materials science," Calphad, vol. 26, pp. 273-312, June 2002.

[36] J.-O. Andersson and J. gren, "Models for numerical treatment of multicomponent diffusion in simple phases," Journal of Applied Physics, vol. 72, pp. 1350-1355, Aug. 1992.

[37] T. Philippe and P. W. Voorhees, "Ostwald ripening in multicomponent alloys," Acta Materialia, vol. 61, pp. 4237-4244, June 2013.

[38] T. Philippe, D. Blavette, and P. W. Voorhees, "Critical nucleus composition in a multicomponent system," The Journal of Chemical Physics, vol. 141, p. 124306, Sept. 2014.

[39] Q. Chen, J. Jeppsson, and J. gren, "Analytical treatment of diffusion during precipitate growth in multicomponent systems," Acta Materialia, vol. 56, pp. 1890-1896, May 2008.

[40] D. Larouche, "A new theory of the solid-state growth of embryos during nucleation: the fundamental role of interfacial mobility," Philosophical Magazine, vol. 98, pp. 2035-2060, Aug. 2018. 